\title{
Level Crossing Rate of Macrodiversity System in the Presence of Multipath Fading and Shadowing
}

\author{
Branimir JAKSIC ${ }^{1}$, Dusan STEFANOVIC ${ }^{2}$, Mihajlo STEFANOVIC ${ }^{1}$, \\ Petar SPALEVIC ${ }^{3}$, Vladeta MILENKOVIC ${ }^{1}$ \\ ${ }^{1}$ Faculty of Electrical Engineering, University of Nis, Aleksandra Medvedeva 14, 18000 Nis, Serbia \\ ${ }^{2}$ College of Applied Technical Sciences, Aleksandra Medvedeva 20, 18000 Nis, Serbia \\ ${ }^{3}$ Faculty of Technical Sciences, University of Pristina, Knjaza Milosa 7, 38220 Kosovska Mitrovica, Serbia \\ branimir.jaksic@pr.ac.rs, dusan.stefanovic@itcentar.rs, misa.profesor@gmail.com, \\ petarspalevic@yahoo.com, vladeta.milenkovic@elfak.ni.ac.rs
}

\begin{abstract}
Macrodiversity system including macrodiversity $S C$ receiver and two microdiversity $S C$ receivers is considered in this paper. Received signal experiences, simultaneously, both, long term fading and short term fading. Microdiversity SC receivers reduce Rayleigh fading effects on system performance and macrodiversity $S C$ receivers mitigate Gamma shadowing effects on system performance. Closed form expressions for level crossing rate of microdiversity SC receivers output signals envelopes are calculated. This expression is used for evaluation of level crossing rate of macrodiversity $S C$ receiver output signal envelope. Numerical expressions are illustrated to show the influence of Gamma shadowing severity on level crossing rate.
\end{abstract}

\section{Keywords}

Macrodiversity selection combining (SC) receiver, Rayleigh multipath fading, Gamma shadowing, level crossing rate, correlation

\section{Introduction}

Small scale fading and large scale fading degrade system performance and limit quality of service (QoS). Received signal experiences multipath fading resulting in signal envelope variation and shadowing resulting in signal envelope power variation. Reflection and refraction of radio wave cause short term fading and large obstacles between transmitter and receiver cause long term fading. It is important to determine how small scale fading and large scale fading affect performance of wireless communication system as outage probability and bit error probability. There are more distributions which can be applied to describe short term signal envelope variation depending on propagation environment. The most used statistical models are Rayleigh, Rician, Nakagami- $m$, Weibull and $\alpha-\mu$ distributions [1]. Rayleigh and Nakagami- $m$ distributions can be applied to describe signal envelope variation in linear non line-of-sight (LOS) multipath fading channel. In line-ofsight multipath fading environment, small scale signal envelope variation can be described by using Rician distribution. Weibull and $\alpha-\mu$ distributions can be used to describe small scale signal envelope variation in nonlinear multipath fading channel dependent on the number of clusters in propagation environment [2].

There are two distributions which can be applied to describe large scale signal envelope power variation in shadowing fading channels. These statistical models are log-normal distribution and Gamma distribution. Log-normal distribution well describes large scale signal envelope power variation but it does not lead to closed form expression for probability density function of output signal envelope. The expression for outage probability of wireless communication system subjected to long term fading has a closed form, when Gamma distribution describes signal envelope power variation.

Macrodiversity system has one macrodiversity receiver and two or more microdiversity receivers. Macrodiversity receiver reduces large scale fading effects on outage probability and microdiversity receivers reduce small scale fading effects on outage probability. Signal envelope at output of macrodiversity receiver is equal to signal envelope at output of microdiversity receiver with greater signal envelope power at inputs [3].

In this paper macrodiversity selection combining (SC) receiver with two microdiversity SC receivers is considered. There are several combining techniques that can be used to mitigate the influence long term fading and short term fading on system performance. The most frequently combining techniques are maximal ratio combining (MRC), equal gain combining (EGC) and selection combining (SC). MRC receiver enables the best performance and it has the highest implementation complexity. The MRC receiver requires channel state information on each diversity branch and need provide receiver train on each diversity branch. The EGC provides performance comparable to $\mathrm{MRC}$ and it has lower implementation complexity than the MRC receiver. The SC receiver has the least im- 
plementation complexity due to the processing being performed only on one diversity branch. The SC receiver selects and outputs branch with the strongest signal envelope [4-5].

The first order performances of wireless communication systems are outage probability, bit error probability and channel capacity. The second order performances of wireless systems are level crossing rate and average fade duration. Level crossing rate can be calculated as the average value of the first derivative of output signal envelope. Average fade duration can be calculated as a ratio of outage probability and level crossing rate. Outage probability is defined as probability that signal envelope falls below the threshold.

There are more works considering macrodiversity system with correlated branches [6] and level crossing rate of macrodiversity systems [7-9]. In [10], macrodiversity system with macrodiversity SC receiver and two microdiversity MRC receivers are analyzed. Received signal is subjected simultaneously to Nakagami- $m$ multipath fading and Gamma shadowing. Macrodiversity SC receiver is applied to reduce Gamma shadowing and microdiversity MRC receivers are used to reduce Nakagami- $m$ multipath fading. Closed form expressions for average level crossing rate and average fade duration are calculated. In [11], level crossing rate and average fade duration of macrodiversity system with macrodiversity SC receiver and two microdiversity MRC receivers operating over Gamma shadowed Rician multipath fading environment are evaluated.

Macrodiversity system with macrodiversity SC receiver and two microdiversity SC receivers is considered. Received signal experiences short term fading and long term fading resulting in system performance degradation. Microdiversity SC receivers are used to combat Rayleigh short term fading and macrodiversity $\mathrm{SC}$ receiver is used to combat long Gamma term fading. Closed form expression for level crossing rate considering macrodiversity system is calculated. To the best author's knowledge, wireless communication system with macro and micro structures operating over Gamma shadowed Rayleigh multipath fading channels is not reported in open technical literature. Obtained results in this paper can be used in performance analysis and designing macrodiversity systems with macrodiversity SC receiver and two or more microdiversity SC receivers in the presence of Gamma shadowing and Rayleigh multipath fading.

\section{Rayleigh Random Variable Level Crossing Rate}

Squared Rayleigh random variable can be expressed as a sum of two independent, zero mean Gaussian random variables $x_{1}$ and $x_{2}$ with the same variance:

$$
x^{2}=x_{1}^{2}+x_{2}^{2}
$$

where $x$ is Rayleigh random variable. The first derivative of $x$ is

$$
\dot{x}=\frac{1}{x}\left(x_{1} \dot{x}_{1}+x_{2} \dot{x}_{2}\right)
$$

where $\dot{x}_{1}$ and $\dot{x}_{2}$ are independent, zero mean Gaussian random variables. Therefore, $\dot{x}$ is Gaussian random variable as linear transformation of Gaussian random variables. The average value of $\dot{x}$ is:

$$
\overline{\dot{x}}=\frac{1}{x}\left(x_{1} \overline{\dot{x}}_{1}+x_{2} \overline{\dot{x}_{2}}\right) .
$$

The variance of the first derivative of Rayleigh random variable is

$$
\sigma_{\dot{x}}^{2}=\frac{1}{x^{2}}\left(x_{1}^{2} \sigma_{\dot{x}_{1}}^{2}+x_{2}^{2} \sigma_{\dot{x}_{2}}^{2}\right)
$$

where

$$
\sigma_{\dot{x}_{1}}^{2}=\sigma_{\dot{x}_{2}}^{2}=\pi^{2} f_{m}^{2} \sigma^{2}=f_{1}^{2}
$$

where $f_{m}$ is maximal Doppler frequency and $f_{1}$ is normalized Doppler frequency. After substituting (5) in (4), the expression for variance becomes:

$$
\sigma_{\dot{x}}^{2}=\frac{f_{1}^{2}}{x^{2}}\left(x_{1}^{2}+x_{2}^{2}\right)=f_{1}^{2} .
$$

The probability density function of $\dot{x}$ is

$$
p_{\dot{x}}(\dot{x})=\frac{1}{\sqrt{2 \pi} f_{1}} e^{-\frac{\dot{x}^{2}}{2 f_{1}^{2}}} .
$$

Rayleigh random variable and the first derivative of Rayleigh random variable are independent. Therefore, joint probability density function of Rayleigh random variable and the first derivative of Rayleigh random variable is

$$
p_{x \dot{x}}(x \dot{x})=p_{x}(x) p_{\dot{x}}(\dot{x})=\frac{2 x}{\Omega} e^{-\frac{x^{2}}{\Omega}} \frac{1}{\sqrt{2 \pi} f_{1}} e^{-\frac{\dot{x}^{2}}{2 f_{1}^{2}}}
$$

The level crossing rate of Rayleigh random variable can be calculated as average value of the first derivative of Rayleigh random variable:

$$
\begin{aligned}
& N_{x}=\int_{0}^{\infty} p_{x \dot{x}}(x \dot{x}) \cdot \dot{x} \cdot d \dot{x} \\
& =\frac{2 x}{\Omega} e^{-\frac{x^{2}}{\Omega}} \int_{0}^{\infty} \dot{x} \frac{1}{\sqrt{2 \pi} f_{1}} e^{-\frac{\dot{x}^{2}}{2 f_{1}^{2}}} d \dot{x}=\frac{2 x}{\Omega} e^{-\frac{x^{2}}{\Omega}} f_{1}
\end{aligned}
$$

The expression for level crossing rate of Rayleigh random variable can be used in performance analysis of wireless communication system operating over Rayleigh multipath fading environment. 


\section{Level Crossing Rate of SC Receiver Output Signal Envelope}

The average level crossing rate (LCR) is a measure [12] that clearly reflects the performances of fading affected system and is used for modeling of wireless communication systems. LCR is related to criterion used to assess error probability of packets of distinct length [4], and to determinate parameters of equivalent channel, modeled by a Markov chain with defined number of states. LCR is used for determining of the rate at which the envelope of the received signal crosses a specified defined level.

Double SC receiver operating over Rayleigh multipath fading channel is considered. Signal envelopes at inputs of SC receiver are denoted with $x_{1}$ and $x_{2}$ and signal envelope at output of $\mathrm{SC}$ receiver is denoted with $x$. At inputs of SC receiver identical and independent Rayleigh multipath fading is present. Probability density function of $\mathrm{SC}$ receiver output signal is

$$
\begin{aligned}
p_{x}(x) & =p_{x_{1}}(x) F_{x_{2}}(x)+p_{x_{2}}(x) F_{x_{1}}(x) \\
& =2 p_{x_{1}}(x) F_{x_{2}}(x)
\end{aligned}
$$

where $F_{x 2}(x)$ is cumulative distribution function of Rayleigh random variable:

$$
F_{x_{2}}(x)=1-e^{-\frac{x^{2}}{\Omega}}
$$

After substituting (11) in (10), the expression for $p_{x}(x)$ becomes

$$
p_{x}(x)=\frac{2 x}{\Omega} e^{-\frac{x^{2}}{\Omega}}\left(1-e^{-\frac{x^{2}}{\Omega}}\right)
$$

where $\Omega$ is signal envelope power.

The joint probability density function of $x$ and $\dot{x}$ is

$$
\begin{aligned}
p_{x \dot{x}}(x \dot{x}) & =p_{x_{1} \dot{x}_{1}}(x \dot{x}) F_{x_{2}}(x)+p_{x_{2} \dot{x}_{2}}(x \dot{x}) F_{x_{1}}(x) \\
& =2 p_{x_{1} \dot{x}_{1}}(x \dot{x}) F_{x_{2}}(x) \\
& =\frac{2 x}{\Omega} e^{-\frac{x^{2}}{\Omega}}\left(1-e^{\left.-\frac{x^{2}}{\Omega}\right)} \frac{1}{\sqrt{2 \pi} f_{1}} e^{-\frac{\dot{x}^{2}}{2 f_{1}^{2}}} .\right.
\end{aligned}
$$

Level crossing rate of SC receiver output signal envelope is

$$
\begin{aligned}
N_{x} & =\int_{0}^{\infty} p_{x x}(x \dot{x}) \cdot \dot{x} \cdot d \dot{x} \\
& =\frac{2 x}{\Omega} e^{-\frac{x^{2}}{\Omega}}\left(1-e^{-\frac{x^{2}}{\Omega}} \int_{0}^{\infty} \dot{x} \frac{1}{\sqrt{2 \pi} f_{1}} e^{-\frac{\dot{x}^{2}}{2 f_{1}^{2}}} d \dot{x}\right. \\
& =\frac{2 x f_{1}}{\Omega} e^{-\frac{x^{2}}{\Omega}}\left(1-e^{-\frac{x^{2}}{\Omega}}\right) .
\end{aligned}
$$

The expression for level crossing rate can be applied for calculation of average fade duration of wireless communication system with dual SC receiver operating over Gamma shadowed Rayleigh multipath fading channels.

\section{Level Crossing Rate of Macrodiversity SC Receiver Output Signal Envelope}

Macrodiversity system with macrodiversity SC receiver and two microdiversity SC receivers operating over shadowed multipath fading environment is considered. Short term Rayleigh fading and Gamma correlated long term fading are presented at inputs of microdiversity SC receivers. Macrodiversity SC receiver reduces signal envelope power variation and microdiversity SC receivers reduce signal envelope variation on system performance. Signal envelopes at output of microdiversity receivers are denoted with $x_{1}$ and $x_{2}$ and macrodiversity SC receiver output signal envelope is denoted with $x$.

Signal envelopes powers at inputs in microdiversity receivers are correlated. Signal envelope powers $\Omega_{1}$ and $\Omega_{2}$ follow Gamma distribution [11]:

$$
\begin{aligned}
& p_{\Omega_{1} \Omega_{2}}\left(\Omega_{1} \Omega_{2}\right)=\frac{1}{\Gamma(c)\left(1-\rho^{2}\right) \rho^{\frac{c-1}{2}} \Omega_{0}^{c+1}} \times \\
& \left(\Omega_{1} \Omega_{2}\right)^{\frac{c-1}{2}} e^{-\frac{\Omega_{1}+\Omega_{2}}{\Omega_{0}\left(1-\rho^{2}\right)}} I_{c-1}\left(\frac{2 \rho}{\Omega_{0}\left(1-\rho^{2}\right)}\left(\Omega_{1} \Omega_{2}\right)^{\frac{1}{2}}\right)
\end{aligned}
$$

where $c$ is fading severity, $\rho$ is correlation coefficient and $\Omega_{0}$ is average power of $\Omega_{1}$ and $\Omega_{2}\left(\Omega_{1} \geq 0, \Omega_{2} \geq 0\right)$. Level crossing rate of signal envelopes $x_{1}$ and $x_{2}$ can be calculated by using expression (14).

The level crossing rate of macrodiversity $\mathrm{SC}$ receiver output signal envelope is

$$
\begin{aligned}
N_{x}= & \int_{0}^{\infty} d \Omega_{1} \int_{0}^{\Omega_{1}} d \Omega_{2} N_{x_{1} / \Omega_{1}} p_{\Omega_{1} \Omega_{2}}\left(\Omega_{1} \Omega_{2}\right) \\
& \quad+\int_{0}^{\infty} d \Omega_{2} \int_{0}^{\Omega_{2}} d \Omega_{1} N_{x_{2} / \Omega_{1}} p_{\Omega_{1} \Omega_{2}}\left(\Omega_{1} \Omega_{2}\right) \\
= & 2 \int_{0}^{\infty} d \Omega_{1} \int_{0}^{\Omega_{1}} d \Omega_{2} N_{x_{1} / \Omega_{1}} p_{\Omega_{1} \Omega_{2}}\left(\Omega_{1} \Omega_{2}\right) \\
= & \frac{\Gamma(c)\left(1-\rho^{2}\right) \rho^{\frac{c-1}{2}} \Omega_{0}^{c+1}}{i=0}\left(\frac{\rho}{\Omega_{0}\left(1-\rho^{2}\right)}\right)^{c+2 i-1} \\
& \times \frac{\left(\Omega_{0}\left(1-\rho^{2}\right)\right)^{c+i}}{\Gamma(c+i) i !} \int_{0}^{\infty} d \Omega_{1} \Omega_{1}^{c+i-2} e^{-\frac{x^{2}}{\Omega_{1}}} e^{-\frac{\Omega_{1}}{\Omega_{0}\left(1-\rho^{2}\right)}} \\
& \times\left(1-e^{-\frac{x^{2}}{\Omega_{1}}}\right) \gamma\left(c+i, \frac{\Omega_{1}}{\Omega_{0}\left(1-\rho^{2}\right)}\right)
\end{aligned}
$$


The incomplete Gamma function $\gamma(n, x)$ is [13]:

$$
\begin{aligned}
\gamma(n, x) & =\Gamma(n)-\frac{1}{n} x^{n} e^{-x}{ }_{1} F_{1}(1, n+1, x) \\
& =\Gamma(n)-\frac{1}{n} x^{n} e^{-x} \sum_{i=0}^{\infty} \frac{x^{i}}{(n+1)_{i}}
\end{aligned}
$$

where $(a)_{n}$ denotes the Pocchammer symbol.

After substituting (17) in (16), the expression for level crossing rate of macrodiversity $\mathrm{SC}$ receiver output signal envelope becomes:

$$
\begin{aligned}
N_{x}= & \frac{4 f_{1} x}{\Gamma(c)\left(1-\rho^{2}\right) \rho^{\frac{c-1}{2}} \Omega_{0}^{c+1}} \sum_{i=0}^{\infty}\left(\frac{\rho}{\Omega_{0}\left(1-\rho^{2}\right)}\right)^{c+2 i-1} \\
& \times \frac{\left(\Omega_{0}\left(1-\rho^{2}\right)\right)^{c+i}}{\Gamma(c+i) i !}\left(I_{1}-I_{2}-I_{3}+I_{4}\right)
\end{aligned}
$$

where

$$
\begin{gathered}
I_{1}=\int_{0}^{\infty} d \Omega_{1} \Omega_{1}^{c+i-2} e^{-\frac{x^{2}}{\Omega_{1}}-\frac{\Omega_{1}}{\Omega_{0}\left(1-\rho^{2}\right)}} \Gamma(c+i), \\
I_{2}=\int_{0}^{\infty} d \Omega_{1} \Omega_{1}^{c+i-2} e^{-\frac{x^{2}}{\Omega_{1}}-\frac{2 \Omega_{1}}{\Omega_{0}\left(1-\rho^{2}\right)}} \frac{1}{c+i}\left(\frac{\Omega_{1}}{\Omega_{0}\left(1-\rho^{2}\right)}\right)^{c+i} \\
\times \sum_{j=0}^{\infty}\left(\frac{\Omega_{1}}{\Omega_{0}\left(1-\rho^{2}\right)}\right)^{j} \frac{1}{(c+i+1)_{j}}, \\
I_{3}=\int_{0}^{\infty} d \Omega_{1} \Omega_{1}^{c+i-2} e^{-\frac{2 x^{2}}{\Omega_{1}}-\frac{\Omega_{1}}{\Omega_{0}\left(1-\rho^{2}\right)}} \Gamma(c+i)
\end{gathered}
$$

and

$$
\begin{aligned}
I_{4} & =\int_{0}^{\infty} d \Omega_{1} \Omega_{1}^{c+i-2} e^{-\frac{2 x^{2}}{\Omega_{1}}-\frac{2 \Omega_{1}}{\Omega_{0}\left(1-\rho^{2}\right)}} \frac{1}{c+i}\left(\frac{\Omega_{1}}{\Omega_{0}\left(1-\rho^{2}\right)}\right)^{c+i} \\
& \times \sum_{j=0}^{\infty}\left(\frac{\Omega_{1}}{\Omega_{0}\left(1-\rho^{2}\right)}\right)^{j} \frac{1}{(c+i+1)_{j}} .
\end{aligned}
$$

After processing and solving integrals (19), (20), (21) and (22), expression (18) becomes:

$$
\begin{aligned}
N_{x} & =\frac{4 f x_{1}}{\Gamma(c)\left(1-\rho^{2}\right) \rho^{\frac{c-1}{2}} \Omega_{0}^{c+1}} \\
& \times \sum_{i=0}^{\infty}\left(\frac{\rho}{\Omega_{0}\left(1-\rho^{2}\right)}\right)^{c+2 i-1} \frac{\left(\Omega_{0}\left(1-\rho^{2}\right)\right)^{c+i}}{\Gamma(c+i) i !}
\end{aligned}
$$

$$
\begin{aligned}
& \times\left[\Gamma(c+i)\left(x^{2} \Omega_{0}\left(1-\rho^{2}\right)\right)^{\frac{c+i-1}{2}}\right. \\
& \times K_{c+i-1}\left(2 \sqrt{\frac{x^{2}}{\Omega_{0}\left(1-\rho^{2}\right)}}\right)- \\
& -\frac{1}{c+i} \sum_{j=0}^{\infty} \frac{1}{(c+i+1)_{j}} \frac{1}{\left(\Omega_{0}\left(1-\rho^{2}\right)\right)^{c+i+j}} \\
& \times\left(\frac{1}{2} x^{2} \Omega_{0}\left(1-\rho^{2}\right)\right)^{c+i+\frac{j}{2}-\frac{1}{2}} \\
& \times K_{2 c+2 i+j-1}\left(2 \sqrt{\frac{2 x^{2}}{\Omega_{0}\left(1-\rho^{2}\right)}}\right)- \\
& -\Gamma(c+i)\left(2 x^{2} \Omega_{0}\left(1-\rho^{2}\right)\right)^{\frac{c+i-1}{2}} \\
& \times K_{c+i-1}\left(2 \sqrt{\frac{2 x^{2}}{\Omega_{0}\left(1-\rho^{2}\right)}}\right)+ \\
& +\frac{1}{c+i} \sum_{j=0}^{\infty} \frac{1}{(c+i+1)_{j}} \frac{1}{\left(\Omega_{0}\left(1-\rho^{2}\right)\right)^{c+i+j}} \\
& \times\left(x^{2} \Omega_{0}\left(1-\rho^{2}\right)\right)^{c+i+\frac{j}{2}-\frac{1}{2}} \\
& \left.\times K_{2 c+2 i+j-1}\left(2 \sqrt{\frac{4 x^{2}}{\Omega_{0}\left(1-\rho^{2}\right)}}\right)\right]
\end{aligned}
$$

where $K_{n}(x)$ is modified Bessel function of the second kind, order $n$ and argument $x$.

\section{Numerical Results}

In Fig. 1 and Fig. 2, normalized average level crossing rate of macrodiversity SC receiver output signal envelope versus macrodiversity SC receiver output signal envelope is plotted. For lower values of SC receiver output signal envelope, average level crossing rate increases as signal envelope increases and for higher values of signal envelope, level crossing rate decreases as signal envelope increases. The influence of signal envelope on level crossing rate is greater for higher values of signal envelope. Level crossing rate decreases as Gamma fading severity increases. For higher values of Gamma shadowing severity, long term fading is of less severity. Graphical results are shown; average level crossing rate increases as correlation coefficient increases. Diversity gain decreases as correlation coefficient increases. 


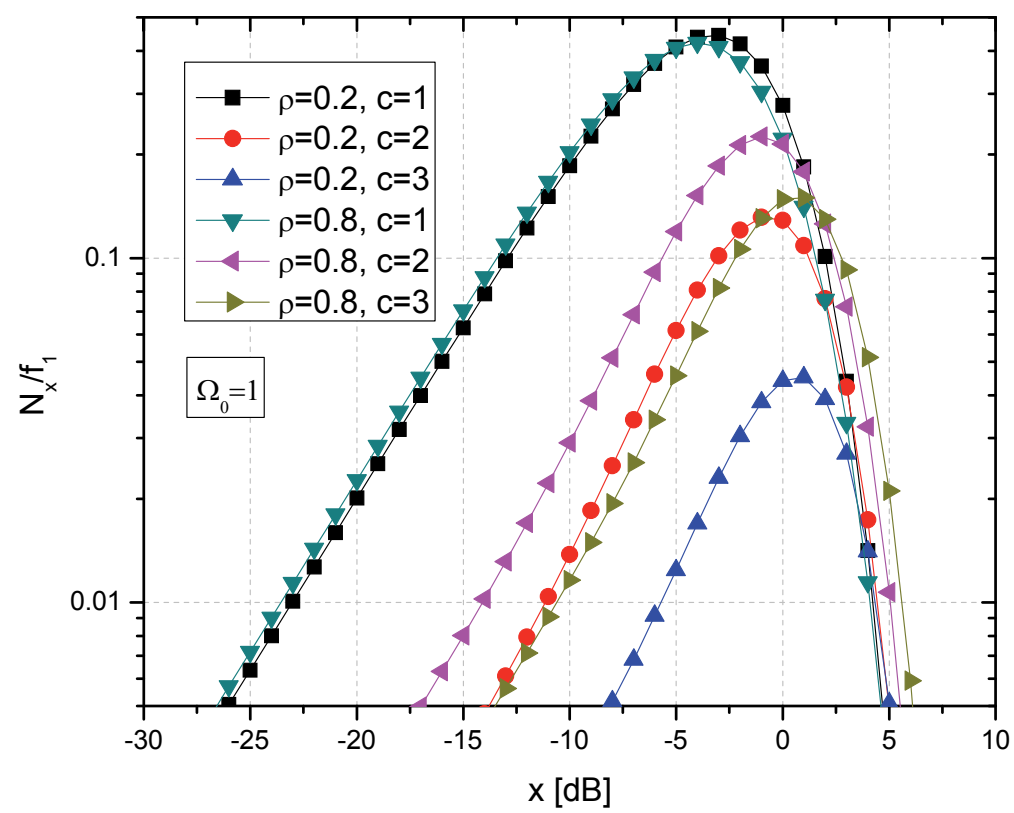

Fig. 1. Level crossing rate of macrodiversity SC receiver output signal envelope for different values of Gamma shadowing severity parameter c.

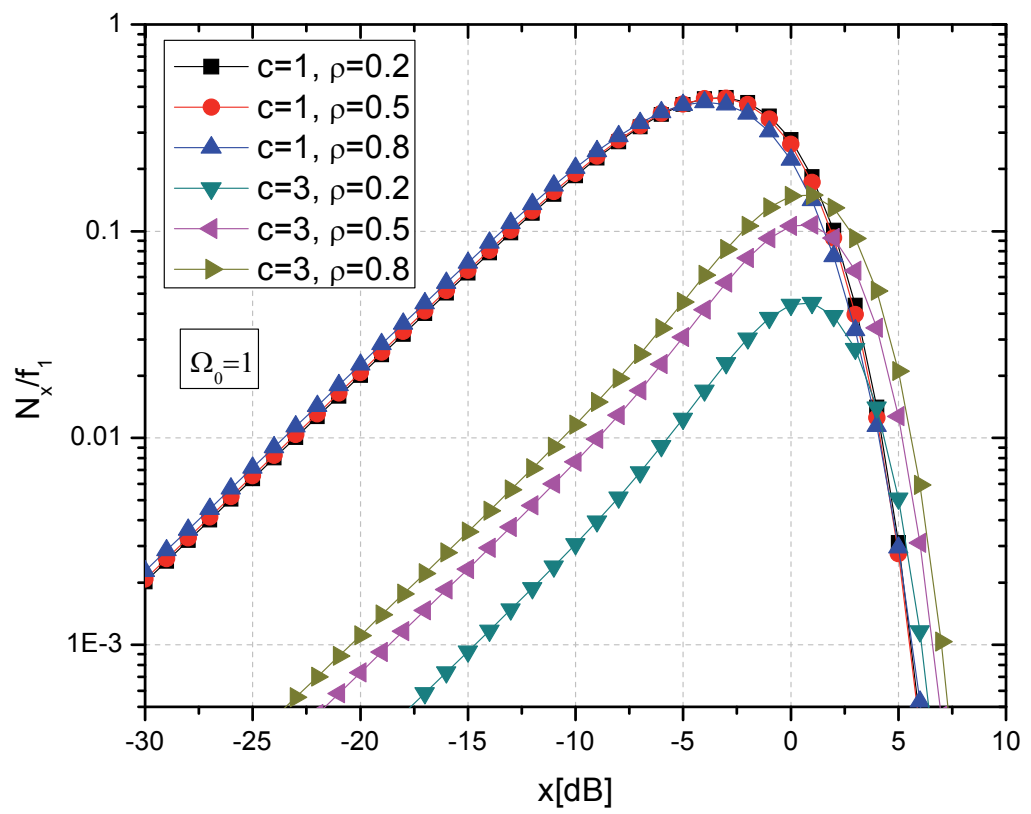

Fig. 2. Level crossing rate of macrodiversity SC receiver output signal envelope for different values of correlation coefficient $\rho$.

In Fig. 3, normalized average level crossing rate of macrodiversity SC receiver output signal envelope versus Gamma shadowing severity parameter $c$ for several values of correlation coefficient is plotted. Average level crossing rate decreases as Gama fading severity increases. When Gamma fading severity parameter $c$ goes to infinity composite Gamma shadowed, Rayleigh multipath channel goes to Rayleigh multipath fading channel.

Normalized average level crossing rate of macrodiversity SC receiver output signal envelope versus correlation coefficient $\rho$ for several values of Gamma shadowing severity is illustrated in Fig. 4. Average level crossing rate increases as coefficient of correlation increases. Outage probability is better for lower values of average level crossing rate. When correlation coefficient goes to 1 , level crossing rate has maximum and system performance is the worst. For the case, the least value of signal envelope occurs at both antennas.

\section{Conclusion}

Macrodiversity system with macrodiversity SC receiver and two microdiversity $\mathrm{SC}$ receivers subjected simultaneously to Rayleigh multipath fading and Gamma shadowing has been analyzed in this paper. Closed form 


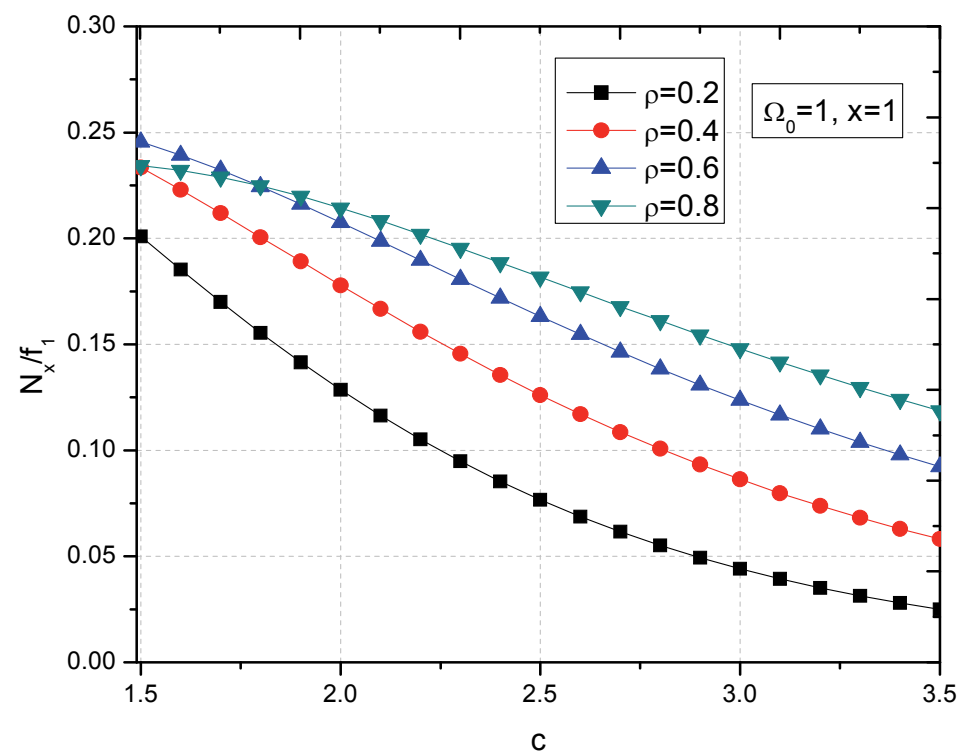

Fig. 3. Level crossing rate of macrodiversity SC receiver output signal envelope versus Gamma shadowing severity parameter c.

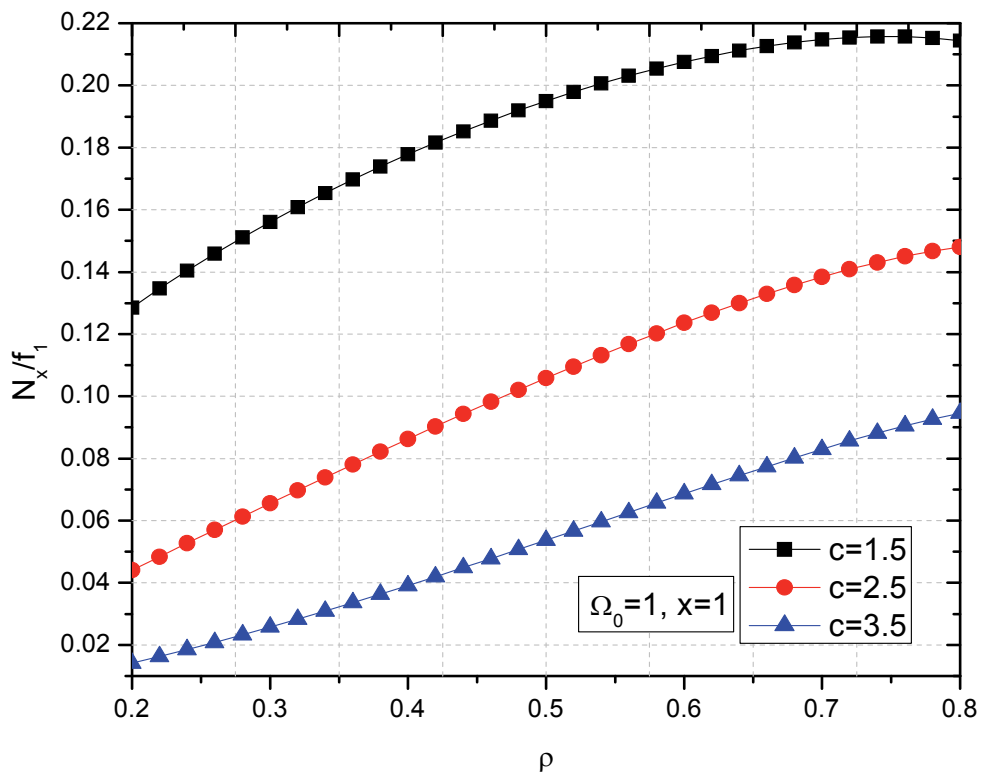

Fig. 4. Level crossing rate of macrodiversity SC receiver output signal envelope versus correlation coefficient $\rho$.

expressions for LCR of macrodiversity SC receiver output signal envelope are derived. Capitalizing on obtained expressions numerical results are presented graphically to show Gamma shadowing severity effects and correlation coefficient effects on LCR of macrodiversity system output signal envelope. LCR reduction has been analyzed as a function of Gamma fading severity decrease and correlation coefficient decrease.

Presented performance evaluation for proposed communication scenarios provides insight into the performance dependence on key system parameters. Presented LCR analysis allows system designers to perform trade-off studies among the various communication type/drawback combinations in order to determine the optimal choice in the presence of given constraints.

\section{References}

[1] SUBER, G. L. Mobile Communication. 2nd ed. Dordrecht: Kluwer Academic Publisher, 2003.

[2] PROAKIS, J. Digital Communications. $4^{\text {nd }}$ ed. New York: McGraw-Hill, 2001.

[3] MUKHERJEE, S., AVIDOR, D. Effect of microdiversity and correlated macrodiversity on outages in a cellular system. IEEE Transactions on Wireless Technology, 2003, vol. 2, no. 1, p. 50 to 58. DOI: $10.1109 /$ TWC.2002.806363

[4] PANIC, S., STEFANOVIC, M., ANASTASOV, J., SPALEVIC, P. Fading and Interference Mitigation in Wireless Communications. USA: CRC Press, 2013.

[5] SIMON, M. K., ALOUINI, M. S. Digital Communication over Fading Channels. USA: John Wiley \& Sons, 2000. 
[6] SHANKAR, P. M. Analysis of microdiversity and dual channel macrodiversity in shadowed fading channels using a compound fading model. International Journal of Electronics and Communications (AEUE), 2008, vol. 62, no. 6, p. 445-449. DOI: 10.1016/j.aeue.2007.06.008

[7] KLINGENBRUnN, T., MOGENSEN, P. Modelling crosscorrelated shadowing in network simulations. In Proceedings of the 50 $0^{\text {th }}$ IEEE Vehicular Technology Conf. (VTC 1999 Fall). Amsterdam (The Netherlands), 1999, vol. 3, p. 1407-1411. DOI: 10.1109/VETECF.1999.801494

[8] ZHANG, J., AALO, V. Effect of macrodiversity on average-error probabilities in a Rician fading channel with correlated lognormal shadowing. IEEE Transactions on Communications, 2001, vol. 49, no. 1, p. 14-18. DOI: $10.1109 / 26.898244$

[9] SAFAK, A., PRASAD, R. Effects of correlated shadowing signals on channel reuse in mobile radio systems. IEEE Transactions on Vehicular Technology, 1991, vol. 40, no. 4, p. 708-713. DOI: 10.1109/25.108381

[10] STEFANOVIC, D., PANIC, S., SPALEVIC, P. Second-order statistics of SC macrodiversity system operating over Gamma shadowed Nakagami-m fading channels. $A E U$ - International Journal of Electronics and Communications, 2011, vol. 65, no. 5, p. 413-418. DOI: 10.1016/j.aeue.2010.05.001

[11] SEKUlOVIC, N., STEFANOVIC, M. Performance analysis of system with micro- and macrodiversity reception in correlated Gamma shadowed Rician fading channels. Wireless Personal Communications, 2012, vol. 65, no. 1, p. 143-156. DOI: $10.1007 / \mathrm{s} 11277-011-0232-8$

[12] ISKANDER, C. D., MATHIOPOULOS, P. T. Analytical level crossing rate and average fade duration in Nakagami fading channels. IEEE Transactions on Communications, 2002, vol. 50, no. 8, p 1301-1309. DOI: 10.1109/TCOMM.2002.801465

[13] GRADSHTEYN, I., RYZHIK, I. Tables of Integrals, Series, and Products. New York: Academic Press, 1994.

\section{About the Authors ...}

Branimir JAKSIC was born in Kosovska Mitrovica, Serbia, in 1984. He received B.Sc. and M.Sc. degrees in Electrical Engineering from the Faculty of Technical Sciences in Kosovska Mitrovica, University of Pristina, Serbia. He is Ph.D. candidate in the Faculty of Electronic Engineering, University of Nis, Serbia. Areas of research include statis- tical communication theory and optical telecommunications. He has authored several scientific papers on the above subject.

Dusan STEFANOVIC was born in Nis, Serbia, in 1979. He received the M.Sc. in Electrical Engineering from the Faculty of Electronic Engineering (Dept. of Telecommunications), University of Nis, Serbia, in 2005 and Ph.D. in the same department in 2012. He is interested in optical and wireless communication. Now he is working in College of Applied Technical Sciences as professor on the following subjects: Database administration, computer networks, administration of computer networks and optolaser technologies.

Mihajlo STEFANOVIC was born in Nis, Serbia in 1947. He received B.Sc., M.Sc. and Ph.D. degrees in Electrical Engineering from the Faculty of Electronic Engineering (Dept. of Telecommunications), University of Nis, Serbia, in 1971, 1976 and 1979, respectively. His primary research interests are statistical communication theory, optical and satellite communications. He has written or co-authored a great number of journal publications. Dr. Stefanovic is a full-time professor with the Dept. of Telecommunications, Faculty of Electronic Engineering, University of Nis, Serbia.

Petar SPALEVIC was born in Kraljevo, Serbia, in 1973. He received the B.S. degree from the Faculty of Electronic Engineering, University of Pristina, in 1997, and M.S. and Ph.D. degrees from the Faculty of Electronic Engineering, University of Nis in 1999 and 2003, respectively. He is a Professor with the Faculty of Technical Sciences in Kosovska Mitrovica. His primary research interests are statistical communications theory, wireless communications, applied probability theory and optimal receiver design.

Vladeta MILENKOVIC was born in Nis, Serbia. He received B. Sc. and M. Sc. degrees in Electrical Engineering from the Faculty of Electronic Engineering, University of Nis, Serbia. He is Ph.D. candidate in the same faculty. He has authored several scientific papers on wireless communications, metrology and measurement techniques. 